\title{
Image Detection Technology of Implicit Sequence Difference Based on Differential Information Theory
}

\author{
Jinfeng Guo $^{1}$, Jing Shao ${ }^{2}$ \\ ${ }^{1}$ College of Textile and Garment, Hebei University of science and Technology, Shijiazhuang, \\ 050000, China \\ ${ }^{2}$ School of Arts, Hebei University of Science and Technology, Shijiazhuang, 050000, China \\ jinfeng_guo2013@yeah.net
}

Keywords: Emotional openness, Implicit sequence, Curve fitting, Genetic algorithm, RSI test, differences.

\begin{abstract}
Implicit sequence learning paradigm is an important form of implicit learning. In order to study the probability implicit sequence differences of high and low emotion openness, this paper uses stepwise half method to select different RSI points, and each RSI point is done binary coding, finally it uses the genetic algorithm to complete the cross process of sequence. Through the iteration calculation and comparison, this paper obtains the implicit test scores in different RSI conditions for different openness, finally carries on the curve fitting for multiple RSI points scores by using the MATLAB software. From the fitting results it can be found that implicit awareness process for high emotion openness and low emotion openness is different, and implicit learning test result has certain changing trend with RSI, so as to reveal the implicit gradient process difference of high and low emotion openness.
\end{abstract}

\section{Introduction}

Implicit sequence learning is another major research paradigm in implicit learning field besides the artificial grammar learning. In this paradigm, a stimulus symbol sequentially appears in horizontal arrangement several locations, and this order is following a specific sequence [1, 2]. In general, the right stimulation has no consciousness or no affect on the implicit learning of sequence. But the level of emotional open reaction probability is different, and it has obvious difference in implicit sequence. In order to verify the difference, the paper uses genetic algorithm to encode on the on high and low emotion openness, and uses MATLAB to do curve fitting on RSI implicit test scores. The main frame structure is as shown in Figure 1.

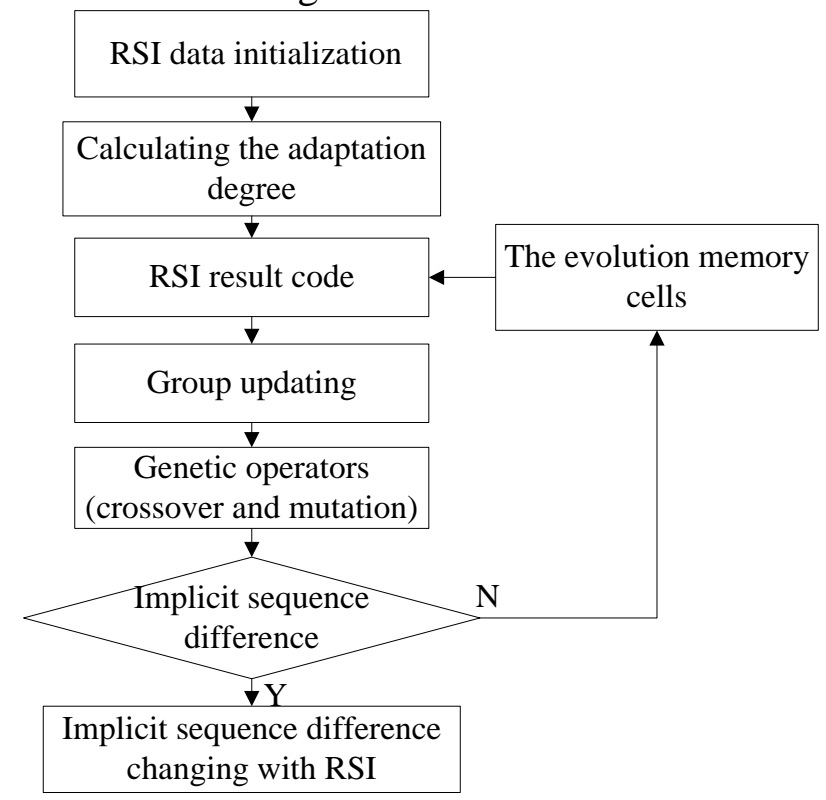

Fig.1: The calculation framework map of implicit learning sequence difference 
Figure 1 shows the calculation framework map of implicit learning sequence difference. The main basis is the data mining of RSI test results, and it uses the genetic algorithm to encode on the RSI different emotion openness test data, using genetic crossover and mutation to get differences of implicit sequence [3]. When the data has difference, it uses MATLAB to fit differences trend; if there is no difference, then it re-calculate the adaptation degree, and uses the genetic algorithm to get the difference of implicit learning sequence.

\section{The Genetic Algorithm Design of RSI Measuring Point Result Code}

Genetic algorithm can use the fitness function to evaluate the RSI test point results, which is as the basis of choice, no need to use external data information [4-6]. In order to study the changing curve of high and low emotion openness for different RSI test results, based on the genetic algorithm, this paper generates a random RSI grade sequence.

$$
\left\{c_{t-1}, c_{t-1}^{1}, c_{t-1}^{2}, \cdots, c_{t-1}^{n}\right\}, c_{t-1} \in R .
$$

$c$ is measuring point score, $\left\{c_{l}^{1}, c_{l}^{2}, \cdots, c_{l}^{n}\right\}$ is as initial chromosome, solving the maximum value of fitness function $u(x)$. RSI value can be expressed as function $f(x)$, and the expression is as follows:

$$
f(x)=\left\{\begin{array}{cc}
u(x)+C_{\min } \text { when } u(x)+C_{\min }>0 \\
0 & \text { other }
\end{array} .\right.
$$

Solving the minimum value of fitness function $u(x)$.

$$
f(x)=\left\{\begin{array}{cc}
C_{\max }-u(x) \text { when } u(x)<C_{\max } \\
0 & \text { other }
\end{array} .\right.
$$

$C_{\min }$ can be input value of RSI measuring point, or the minimum value of $g(x)$ before the $K$ generation, and it also may be a function of population variance [7]. $C_{\max }$ can be an appropriate input values, or the maximum value of $g(x)$ in the evolution process. Population scale is $n$, the fitness value of individual $i$ is $f_{i}$, so the selected probability of $i$ is $P_{s i}$.

$$
P_{s i}=\frac{f_{i}}{\sum_{i=1}^{M} f_{i}} .
$$

Single point crossover is called simple cross. In each RSI test points it randomly selects a point as a cross point, and then pairing individual is at the end of the cross point, mutual exchanging partial chromosomes.

Test point A: $1101 \mid 1010$

Test point B: $\quad 1011 \mid 0101$

Test point A': 11010101

Test point B': 10111010

In order to realize the algorithm and do curve fitting on RSI scores, this paper adopts MATLAB genetic algorithm and the curve fitting toolbox to program the algorithm, and the main procedures are as follows:

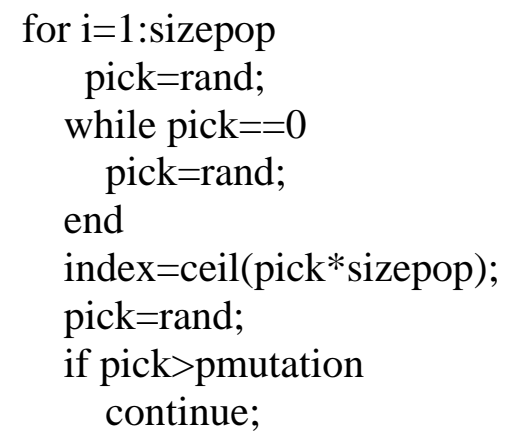




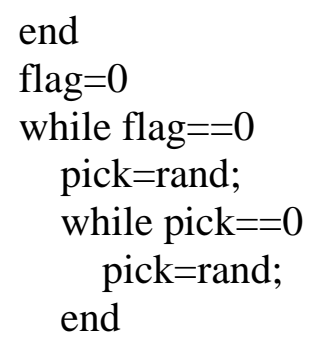

\section{Implicit Learning Differences Sequence Numerical Calculation of High and Low Emotion Openness}

In order to effectively verify the effectiveness and reliability of the RSI measuring point performance evaluation genetic algorithm designed in section second, this paper uses MATLAB genetic algorithm and the curve fitting toolbox to program the algorithm, and the MATLAB toolbox is as shown in Figure 2

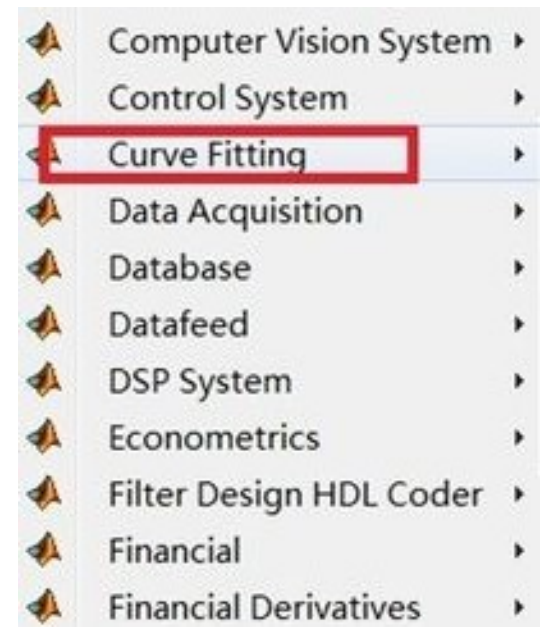

Fig.2: Schematic diagram of MATLAB toolbox

As shown in Figure 2 it represents the MATLAB toolbox. The Simulink is an integrated environment to realize the dynamic system modeling, simulation and analysis, which makes the MATLAB function further extended [8]. It can realize the visualization modeling easily, and the theoretical research is organic combined with engineering practice.

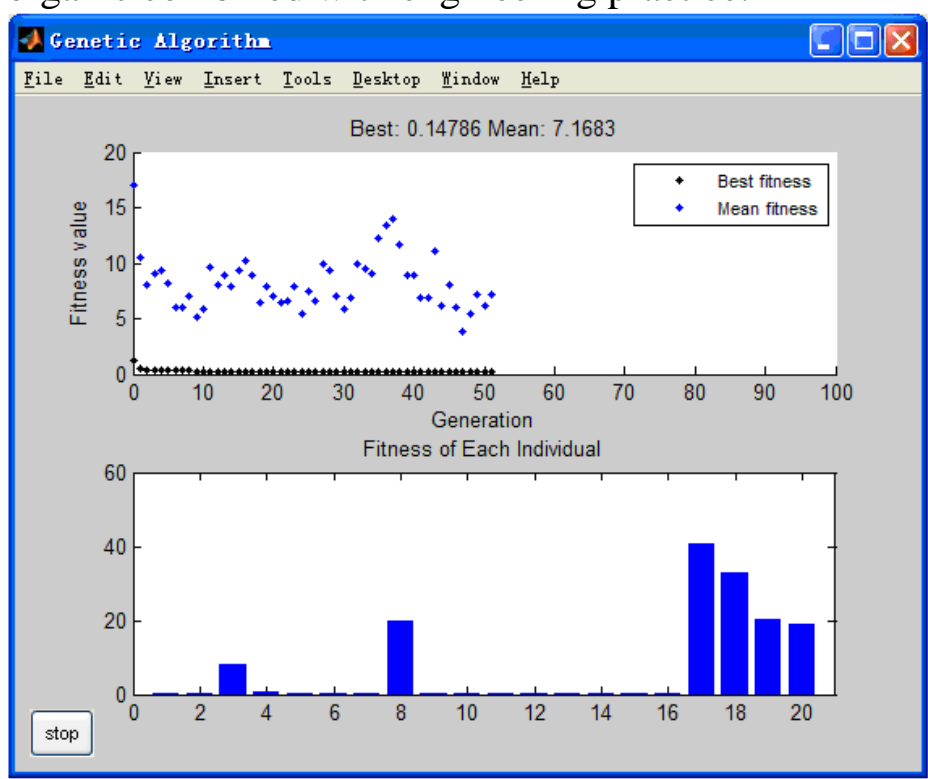

Fig.3: The selection process of RSI genetic algorithm measuring point 
Figure 3 shows the selection process of RSI genetic algorithm measuring point. Through screening the representative test point, it can effectively distinguish the high emotion and low emotion openness [9]. The reaction parameter changing curve of high emotion and low emotion openness on the block is as shown in Figure 4.

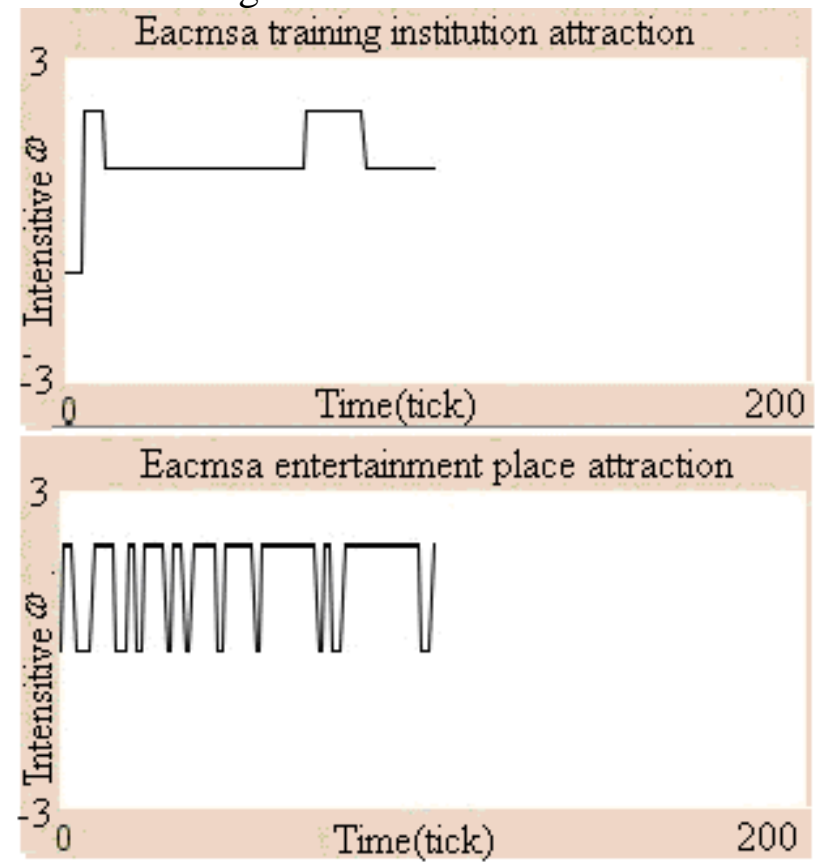

Fig.4: The parameters changing chart of high and low emotion openness

Figure 4 shows the action emotional state and the corresponding intensity changes based on Agent. The figure shows the performance parameters changing caused by target selection and behavior decision [10]. From the chart it can be seen, high and low emotion openness has different reaction on testing block, which is mainly manifested in reaction time, as shown in Table 1.

Table 1: The testing results of high and low emotion openness

\begin{tabular}{lll}
$\begin{array}{l}\text { The test } \\
\text { block }\end{array}$ & $\begin{array}{l}\text { The reaction time of } \\
\text { low emotion } \\
\text { openness (ms) }\end{array}$ & $\begin{array}{l}\text { The reaction time of } \\
\text { high emotion openness } \\
\text { (ms) }\end{array}$ \\
\hline 1 & 450 & 432 \\
2 & 422 & 415 \\
3 & 421 & 413 \\
4 & 415 & 405 \\
5 & 408 & 401 \\
6 & 392 & 380 \\
\hline
\end{tabular}

Table 1 shows RSI test results of high and low emotion openness. From the table it can be seen, the high and low emotion openness has different reaction speed on the test block, which means the implicit sequence has certain differences [11]. In order to show the difference directly, the data will be drawn as the curve as shown in the Figure 5.

Figure 5 represents the test results of high and low emotion openness reaction time [12]. From the chart it can be seen, the reaction time open of high and low emotion openness for different blocks have certain fluctuation, but the reaction time of high emotion openness is faster than that of low emotion openness, and implicit sequence performance is lower that of low emotion openness.

The emotional education belongs to college students' psychological education category; the students are mainly concerned about the things that produce inner experience and emotional reactions. In order to improve the English memory efficiency, the role of emotional education can not be ignored; it can maintain the learning motivation, and learning motivation, thereby reducing the forgotten extent in the English learning process. Therefore, on the basis of research emotional education and English memory efficiency, to present emotional College English memory model 
through theoretical derivation, to analyze the relation between them through the experiment, as Figure 6 shown.

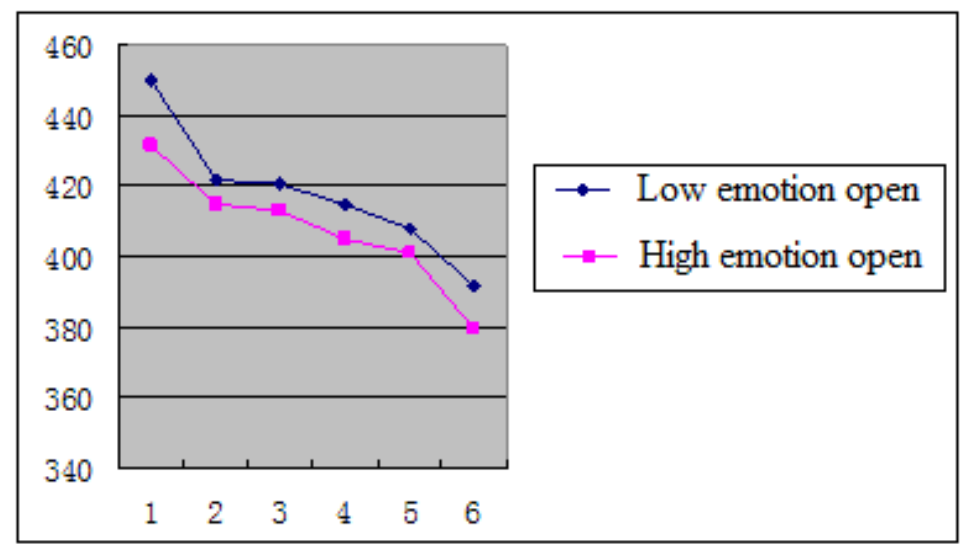

Fig.5: Test results of high and low emotion openness reaction time

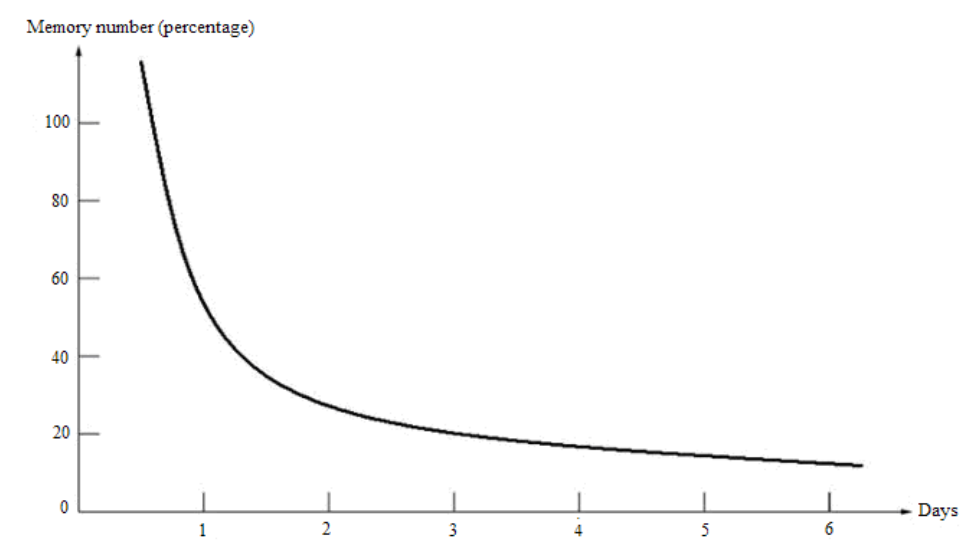

Fig.6: The memory curve

\section{Summary}

This paper uses genetic algorithm to encode the RSI testing result of high and low emotion openness, and uses the genetic algorithm crossover and mutation to mining the difference in implicit sequence data, finally the fitness function of implicit sequence learning paradigm is obtained. This paper use MATLAB to do curve fitting on implicit sequence learning paradigm differences, form the results it can be seen, the high and low emotion openness has different reaction speed on the test block, and the reaction time of high emotion openness is faster than that of low emotion openness, which has certain advantages in implicit sequence.

\section{References}

[1] J. Zhang, B. Zhu, L.L. Liang, M. Hou, Y.L. Teng. Chinese micro-blog emotional recognition and classification based on emotional factors. Journal of Peking University (NATURAL SCIENCE EDITION), 2014, 2(1): 32-35.

[2] W. Gao, Z.Q. Wang, S.S. Li. Study on semi supervised sentiment classification method based on integrated learning. Chinese journal of information, 2013, 4(3): 34-38.

[3] W. Yang, J.J. Song, J.Q. Tang. Subjective and objective sentences analysis in Chinese microblog emotion classification method. Journal of Chongqing University of Technology (NATURAL SCIENCE), 2013, 4(1): 61-64. 
[4] X.J. Zhang, C.L. Zhou. The emotional level BDI subject model research. Pattern recognition and artificial intelligence, 2013, 4(7): 78-81.

[5] Q. Tao, G. Ma, Z.Z. Shi. Expert system reasoning model based on Agent model. Journal of intelligent system, 2013, 5(2): 81-84.

[6] J.Y. Li. Research status and cognitive difficulties of emotion computing. Dialectics of nature, 2012, 5(2): 91-94.

[7] X.C. Pan, Y.F. Wang. Research on Agent structure under emergency response based on BDI. Microcomputer and its application, 2013, 4(5):54-58.

[8] D.L. Zhang, Z.Z. Shi, Y. Pan. The emotional body form model. Pattern recognition and artificial intelligence, 2012, 4(3): 52-56.

[9] J. Shi, Y.F. Wu, L.K. Qiu, X.Q. Lv. Chinese word similarity calculation method based on large scale corpus. Journal of Chinese information, 2013, 4(1): 65-68.

[10] G. Zhang, D.D. Zhou, Q.Q. Ge. Learner characteristics and modeling methods in the adaptive learning system model. Modern educational technology, 2012, 4(5): 12-15.

[11] J.H. Wan. Affective computing. Journal of Shanghai Dian Ji University, 2013, 4(4): 24-28.

[12] S.D. Teng, Z.L. Wang, G.J. Wang. artificial emotion model based on the idea of mental energy. Computer engineering and application, 2012, 4(3): 101-105. 